gerichts war in vielfacher Hinsicht grob rechtswidrig. Dies lag nicht an den (angeblichen) „rechtspositivistischen“ Arbeitsmethoden der beteiligten Richter. Im Gegenteil, eine strikte Orientierung an den Normen der Strafprozessordnung und eine unparteiliche penible Einhaltung rechtsstaatlicher Standards hätten dieses Fehlurteil verhindern können. Unverzichtbar war ferner die gebotene innere Unabhängigkeit der Richter von den Zumutungen außergerichtlicher staatlicher Macht. Hieran fehlte es.

\title{
Helmut Kramer
}

\section{„Wer im Namen des Volkes Recht spricht...“.}

\section{Über Schwierigkeiten bei der Erforschung der Vergangenheit}

Bundesjustizministerin Brigitte Zypries wird nicht müde, ihren Einsatz für die rückhaltlose Aufklärung des nationalsozialistischen Unrechts hervorzuheben und die Bedeutung der „Auseinandersetzung mit Lebensläufen und Karriereentwicklungen" ehemaliger NS-Juristen zu betonen. Wie bei vielen anderen Absichtserklärungen ist allerdings auch hier die Frage, wie weit solche hehren Anliegen in den unteren Ebenen eines Ministeriums umgesetzt werden. $\mathrm{Zu}$ einer strapaziösen Probe aufs Exempel führte die Absicht des Verfassers, für zwei biographische Darstellungen die Personalakten der beiden sowohl der nationalsozialistischen als auch der bundesdeutschen Justiz dienenden Juristen einzusehen. Die Benutzung der Personalakten von Beamten, in diesem Fall der Akten eines furchtbaren Juristen der Hitlerzeit, ist für jeden seriösen Zeitgeschichtsforscher unverzichtbar. Also forderte ich im Bundesjustizministerium die Personalakte Ebersberg an, eines der Komplizen des Massenmordes an „asozialen“ Strafgefangenen. ${ }^{1}$ Wie nicht wenige andere furchtbare Juristen war Ebersberg nach 1945 nämlich nahezu bruchlos in das BMJ übergesiedelt. Die mir nach mehr als drei Monaten und auch dann erst nach Erinnerung erteilte Antwort des BMJ: Personalakten dürften von Privatpersonen (also auch von Wissenschaftlern) nicht eingesehen werden. Allenfalls könnten einzelne Auskünfte erteilt werden, auch dies nur in einem komplizierten Verfahren mit Zustimmung der Hinterbliebenen.

Ich habe mir die Augen gerieben. Wenn der Tod des Amtsträgers 30 Jahre zurückliegt, macht das Bundesarchiv keinerlei Schwierigkeiten. Und auch ich habe seit Jahren volle Einsicht in viele Personalakten mehrerer Landesjustizministerien und Staatsarchive nehmen dürfen.

Den ablehnenden Bescheid hatte das BMJ auf die „beamtenrechtlichen Regelungen (Bundesbeamtengesetz)“ gestützt, ohne jegliche Paragraphenangabe oder sonstige Quelle. Tatsächlich gibt es weder im Bundesbeamtengesetz noch anderswo eine beamtenrechtliche Vorschrift, wonach Personalakten auch für wissenschaftliche Zwecke verschlossen bleiben müssten. Einer Einwilligung dafür bedarf es nur zu Lebzeiten des Beamten; nur insoweit gilt $\$ 90 \mathrm{~d}$ Abs. 2 Bundesbeamtengesetz. Nach dem Tod des Beamten hat der Dienstherr eine Abwägung vorzunehmen: zwischen dem auch nach dem Tod der Person fortwirkenden

1 Vgl. Helmut Kramer, Der Beitrag der Juristen zum Massenmord an Strafgefangenen und die strafrechtliche Ahndung nach 1945, in: Beiträge zur Geschichte der nationalsozialistischen Verfolgung in Norddeutschland, Heft 11 (2009). 
interesse. $^{2}$

Hier musste man dem BMJ mit einer Rechtsbelehrung unter die Arme greifen: Mangels einer weiteren Konkretisierung des Beamtenrechts hätte das BMJ auf die Regelung des modernen Archivrechts in analoger Anwendung zurückgreifen können und müssen, auch abgesehen davon, dass derartige Personalakten der Bundesministerien längst an das Bundesarchiv hätten abgegeben werden müssen (vgl. \90f BBG). Archivgut des Bundes kann, von besonderen Ausnahmefällen abgesehen, grundsätzlich 30 Jahre nach dem Tod der betroffenen Person benutzt werden, eine Voraussetzung, die im Fall des Heinrich Ebersberg (1911-1976) erfüllt war. Übrigens kann bei Personen der Zeitgeschichte und bei Amtsträgern, die in Ausübung ihres Amtes gehandelt haben, ein Anspruch auf Akteneinsicht sogar vor Ablauf der genannten Dreißigjahresfrist bestehen, wegen des in diesen Fällen verstärkten wissenschaftlichen Interesses. Einem besonders begriffsstutzigen Beamten hätte übrigens ein kurzer Blick auf $\int 5$ des Informationsfreiheitsgesetzes auf die Sprünge geholfen.

Nach Erteilung dieses Nachhilfeunterrichtes ging es schnell: Innerhalb von zwei Tagen und nach Andeutung der Möglichkeit einer verwaltungsgerichtlichen Klage erhielt ich die übrigens recht ergiebige Akteneinsicht.

$\mathrm{Zu}$ einem grundsätzlichen Sinneswandel war es im BMJ dennoch nicht gekommen. Gleichzeitig mit dem Antrag in Sachen Ebersberg hatte ich nämlich Einsicht in die Personalakte des Ministerialdirigenten im BMJ Dr. Eduard Dreher beantragt. Dreher ist zwar erst im Jahre 1995 gestorben. Er war aber sowohl „Amtsträger" als auch eine Person der Zeitgeschichte im Sinne von $\ 5$ Abs. 5 Satz 3 Bundesarchivgesetz. Selbst ohne diese besonderen Eigenschaften bedarf es einer Abwägung zwischen dem Schutz der persönlichen Belange des Betroffenen einerseits und dem wissenschaftlichen Forschungsinteresse und dem Interesse der Öffentlichkeit an geschichtlichen und zeitgeschichtlichen Vorgängen andererseits. Am Ergebnis dieser Abwägung konnte in diesem Fall kein Zweifel sein. In den Jahren 1933 bis 1945 hatte Dreher sich durch Erwirkung mehrerer Todesurteile und zahlreiche üble Veröffentlichungen als ideologisch besessener und opportunistischer nationalsozialistischer Jurist hervorgetan. Später im Bundesjustizministerium hatte er u.a. als Generalreferent der Großen Strafrechtskommission die Strafrechtsreform maßgeblich beeinflusst. Sein StGB-Kommentar, der „Dreher“ liegt noch heute (als Fischer, StGB) in der 59. Auflage auf jedem Richtertisch. Dreher wird mit gewichtigen Verdachtsgründen nachgesagt, dass er den $\ 50$ Abs. 2 StGB (heute $\$ 28$ StGB) in das Strafgesetzbuch mit der Folge geschmuggelt hat, dass mit Hilfe der berüchtigten Gehilfenrechtsprechung die Mordtaten von zahlreichen NSG-Tätern rückwirkend verjährt waren.

Nun wieder das bekannte Spiel: Eine Antwort erhielt ich erst nach drei Monaten, auch dies erst nach zweimaliger Erinnerung. Erneut der längst widerlegte unzutreffende Hinweis auf das Bundesbeamtengesetz, obgleich darin allein der innerdienstliche Umgang mit Personalakten geregelt ist. Also bedurfte es einer erneuten Belehrung der Ministerialbeamten über die eindeutige Rechtslage. Auch ein - nach erneut mehr als dreimonatiger Sendepause angebrachtes - an die Bundesjustizministerin Brigitte Zypries persönlich gerichtetes Schreiben brachte nur wenig Bewegung in die Angelegenheit.

Endlich, fast 12 Monate nach der Antragstellung, hat sich das BMJ nun doch zu einer Bewilligung der Akteneinsicht entschlossen. Offensichtlich war man nach Einschaltung mehrerer Abteilungen, Referate und Unterreferate, wohl zu der

2 Vgl. Ulrich Battis, Bundesbeamtengesetz, München 2004, $\$ 90$ f Rn. 8. 
Einsicht gekommen, dass eine Akteneinsichtsverweigerung einer verwaltungsrechtlichen Klage nicht standhalten würde. So arbeitsaufwändig der Kampf um die Personalakte eines furchtbaren Juristen war, ist auf diese Weise doch trotz oder gerade wegen des hartnäckigen Widerstandes des BMJ eine gewisse Klärung über den Zugang zu Personalakten erreicht worden: Bei bedeutenden, in den Bereich der Zeitgeschichte hineinragenden Amtsträgern wird Wissenschaftlern die Akteneinsicht künftig kaum noch verweigert werden können.

Das juristisch mehrfach abgesicherte Ergebnis konnte auch nicht anders ausfallen: Wer im Namen des Volkes Recht spricht, gar „im Namen des Deutschen Volkes“ Unrecht gesprochen oder sonst an dem nationalsozialistischen Unrechtssystem mitgewirkt hat, hat keinen Anspruch darauf, dass sein Name und seine dienstlichen Daten und Handlungen dem Volke vorenthalten werden.

\title{
Peter Derleder
}

\section{Ein Tag im Leben des Aktionariats.}

\author{
Befindlichkeiten und Wahrnehmungshorizonte im Zuge der \\ Wirtschaftskrise
}

Schon früh an diesem Tag Ende Mai ist vor der Frankfurter Messehalle viel Betrieb. Die Organisation einer Hauptversammlung der Deutschen Bank mit bis zu 5.000 Besuchern folgt einem ausgeklügelten, erfahrungsgesättigten Programm mit Sicherheitsdienst, Catering-Unternehmen und allerlei Publikumsservice. Auch die Nichtregierungsorganisationen sind früh dabei, ihre Stände aufzubauen, um die Aktionäre noch rechtzeitig auf all das aufmerksam zu machen, was Klimawandel, Hunger und Massenarmut auf dem Globus erfordern würden. Die angebotenen Flyer dazu finden bei den ankommenden Aktionären nicht gerade reißenden Absatz. Ein unbefangener Beobachter könnte dennoch den Eindruck haben, heute im Zentrum der wirtschaftlichen Macht zu stehen, wo die Weichen für oder gegen eine ökologische oder soziale Wende gestellt werden. Auch die Führungsfiguren der Deutschen Bank, die sich unter den fünf renommiertesten Bankinstituten der Welt sieht, sind rechtzeitig da und weilen schon bei ihren Sitzen auf der Bühne des großen Saals, hinter der sich die finanztechnische Intelligenz des Back Office mit allen Computern eingerichtet hat. Kurz vor Beginn der Sitzung um 10 Uhr bilden sich lange Schlangen vor den Sicherheitsschleusen, die auch dem frustriertesten Akteur den Zugang mit dinglichen Aggressionsinstrumenten verwehren.

Das Publikum weist jedoch ein auffälliges Durchschnittsalter auf. Es dominieren die Siebziger. Viele Paare sind darunter. Sie sind meist auch wenig feierlich gekleidet. Es könnte eine Kaffeefahrt sein. Alle Schichten scheinen vertreten. Man fragt sich unwillkürlich, ob der Einsatz von ein paar Euro für eine einzige Aktie, die zur Teilnahme ausreicht, nicht auch für den Hartz IV-Bezieher lohnend ist, der jeweils unentgeltlich mittags speisen und nachmittags Kaffee und Kuchen zu sich nehmen kann. Für die Speisung der fünftausend gibt es zwar nur Frankfurter Würstchen mit Kartoffelsalat oder wahlweise Tortellini sowie Kaffee, Tee und kleine Eierkuchenstückchen. Es ist auch nach früheren unangenehmen Auftritten dafür Sorge getragen, dass niemand kanisterweise Nahrung abschleppt. Wer un- 